Supporting Information (SI):

\title{
Reduced Graphene Oxide Supported Palladium Nanoparticles for Enhanced Electrocatalytic Activity Towards Formate Electrooxidation in an Alkaline Medium
}

\author{
Vicente Galvan, Dean E. Glass, Amanda F. Baxter, G. K. Surya. Prakash* \\ Loker Hydrocarbon Research Institute, Department of Chemistry, University of \\ Southern California, 837 Bloom Walk, Los Angeles, CA, 90089 \\ *Corresponding Author: gprakash@usc.edu
}




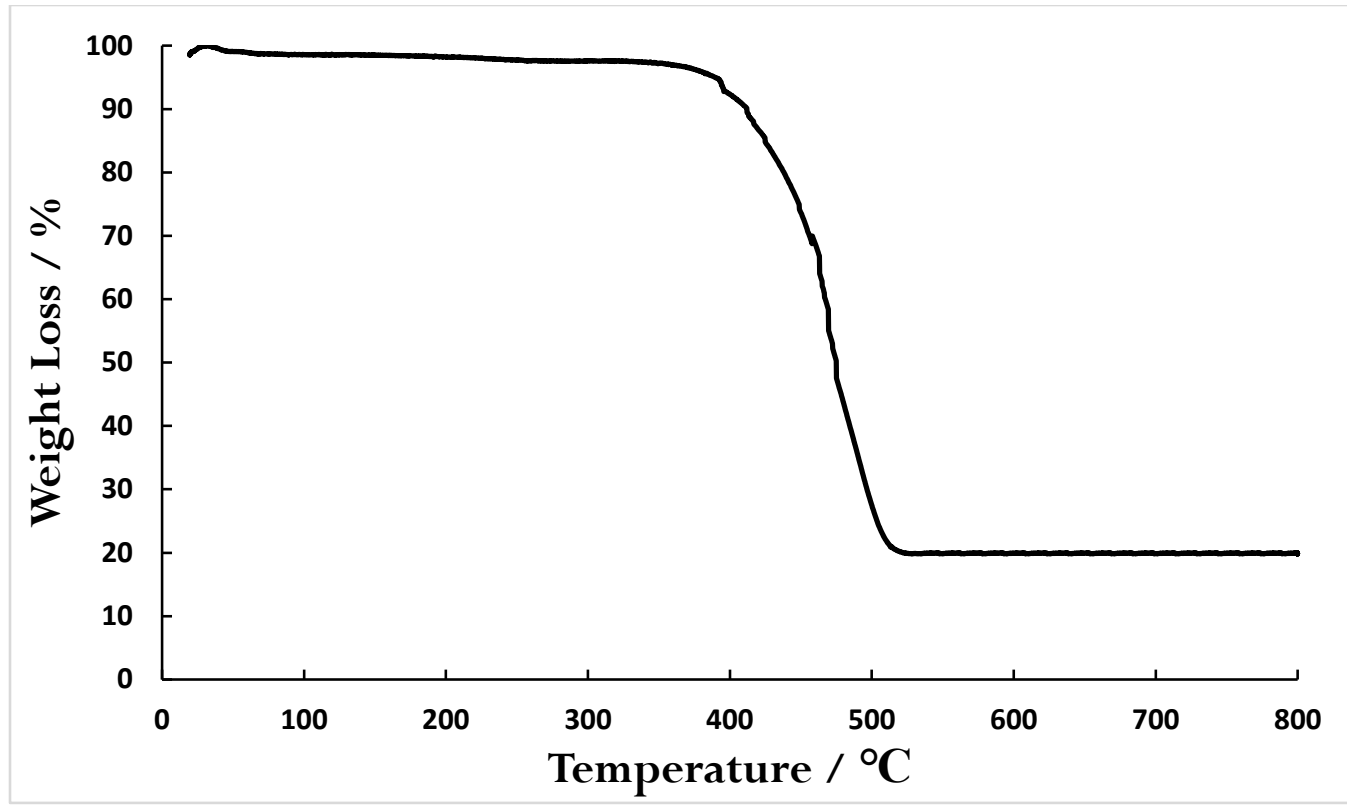

Figure S1. The TGA profile for Pd/rGO catalyst. The TGA experiments were carried out in a $\mathrm{N}_{2}$ atmosphere with a heating rate of $10^{\circ} \mathrm{C} / \mathrm{min}$. 

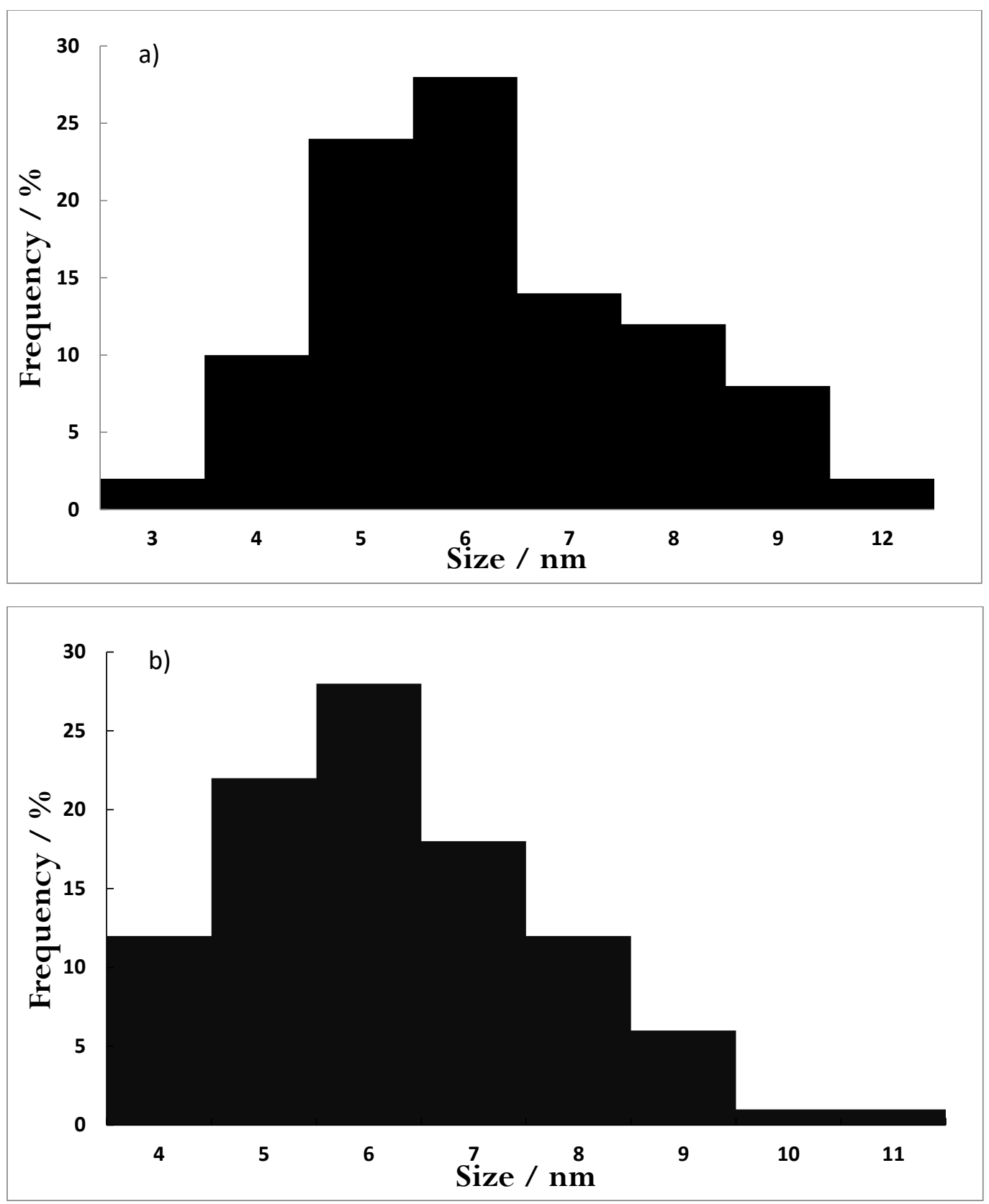

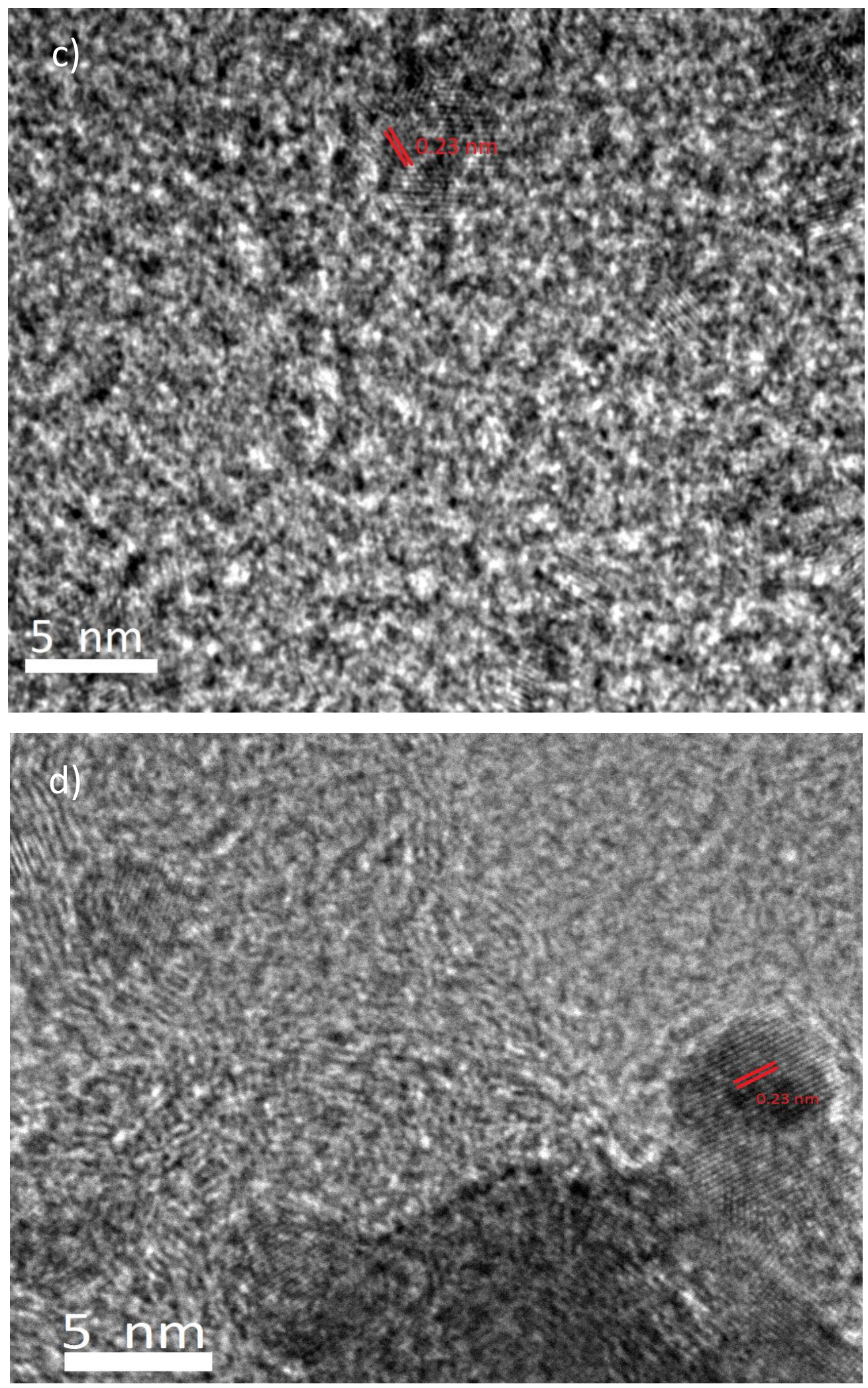

Figure S2. Histogram curves for the catalyst size a) Pd/rGO and b) Pd/C and HRTEM images for c) $\mathrm{Pd} / \mathrm{rGO}$ and d) $\mathrm{Pd} / \mathrm{C}$ 

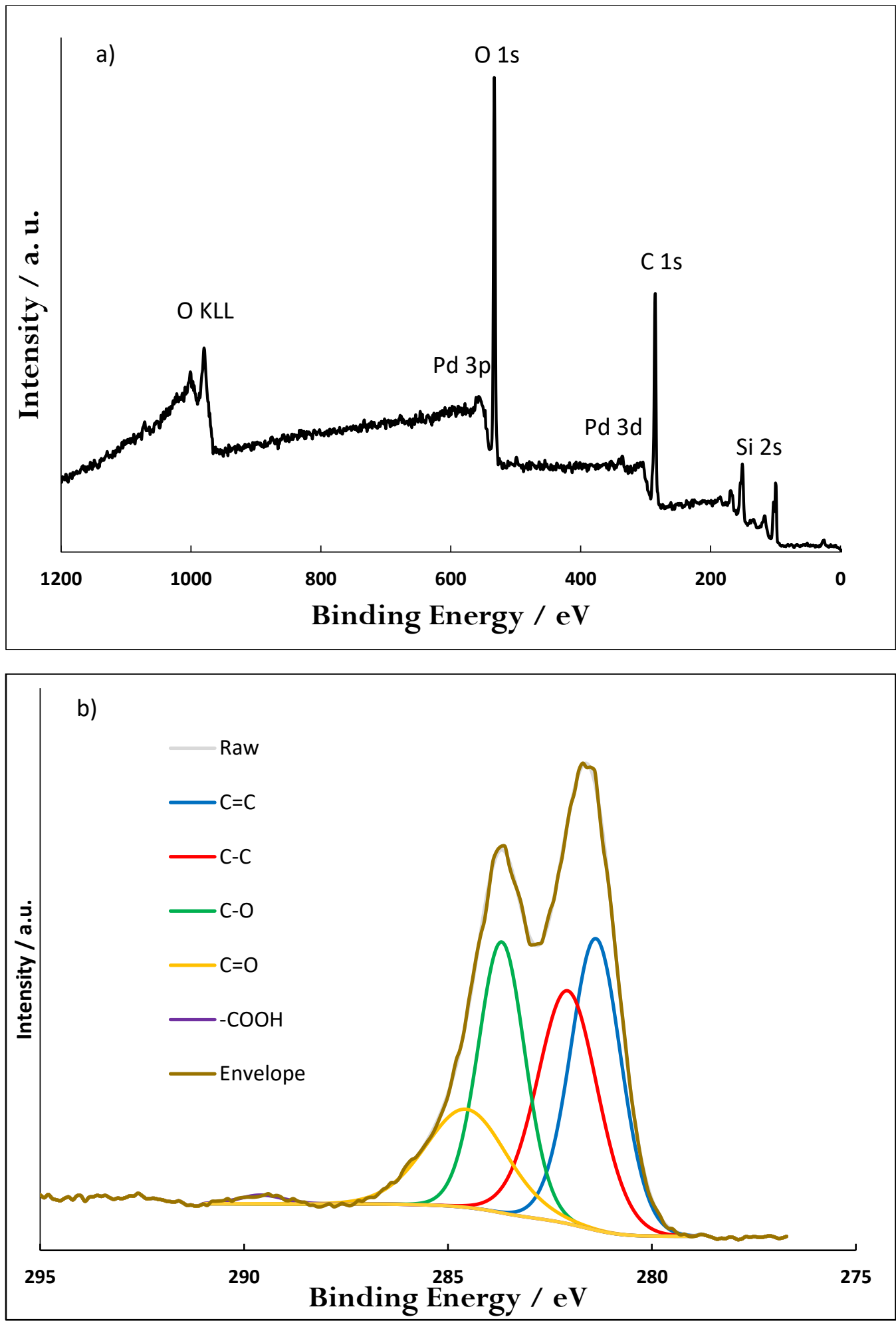

Figure S3. a) XPS survey scan for Pd/rGO and b) XPS C1s spectra of GO. 


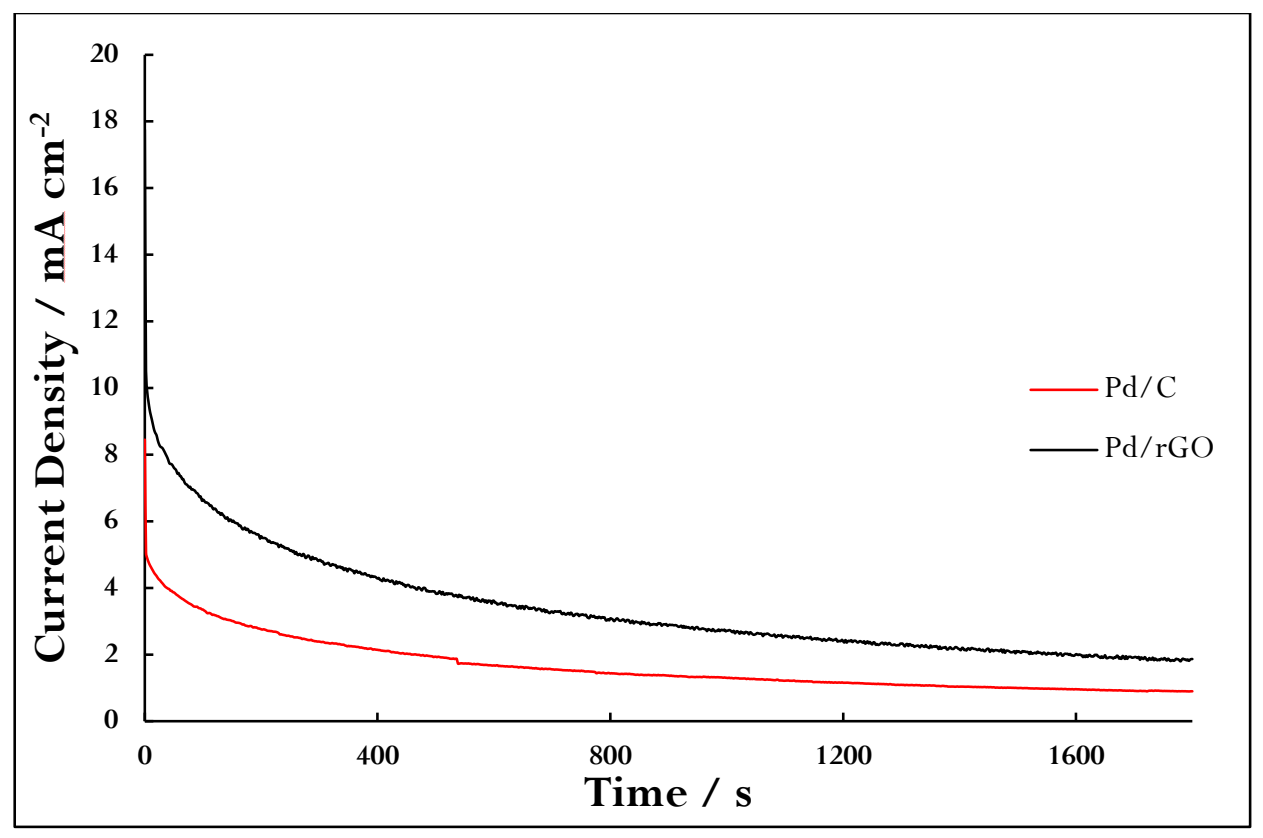

Figure S4. Chronoamperommetry scans of the catalyst at $-0.65 \mathrm{~V}$ vs $\mathrm{Ag} / \mathrm{AgCl}$ at $1000 \mathrm{RPM}$ in 1.0 M HCOONa and $1.0 \mathrm{M} \mathrm{NaOH}$. 

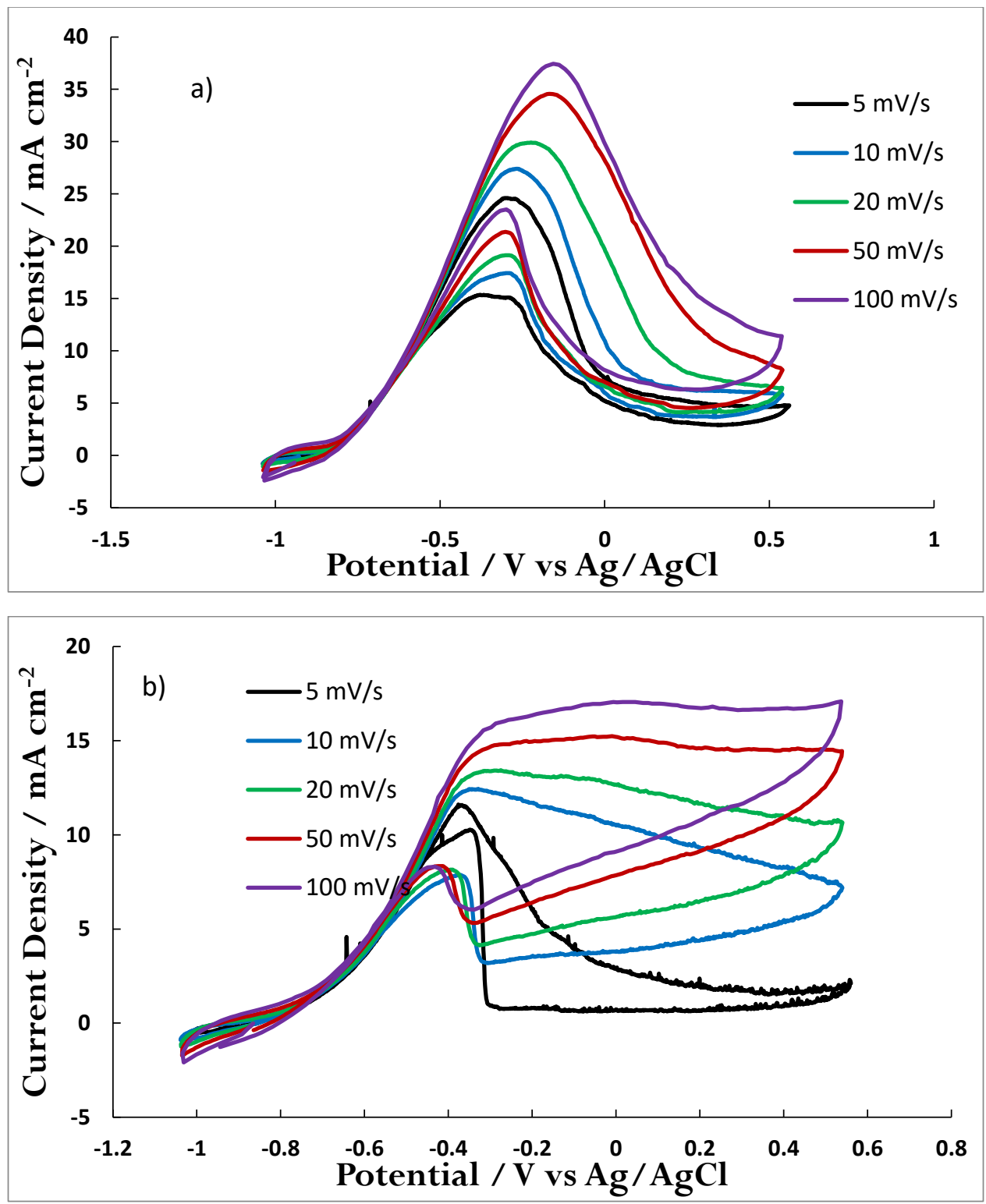

Figure S5. CV scans at different scan rates in $1.0 \mathrm{M}$ HCOONa and $1.0 \mathrm{M} \mathrm{NaOH}$ for: a) $\mathrm{Pd} / \mathrm{rGO}$ and; b) $\mathrm{Pd} / \mathrm{C}$. 

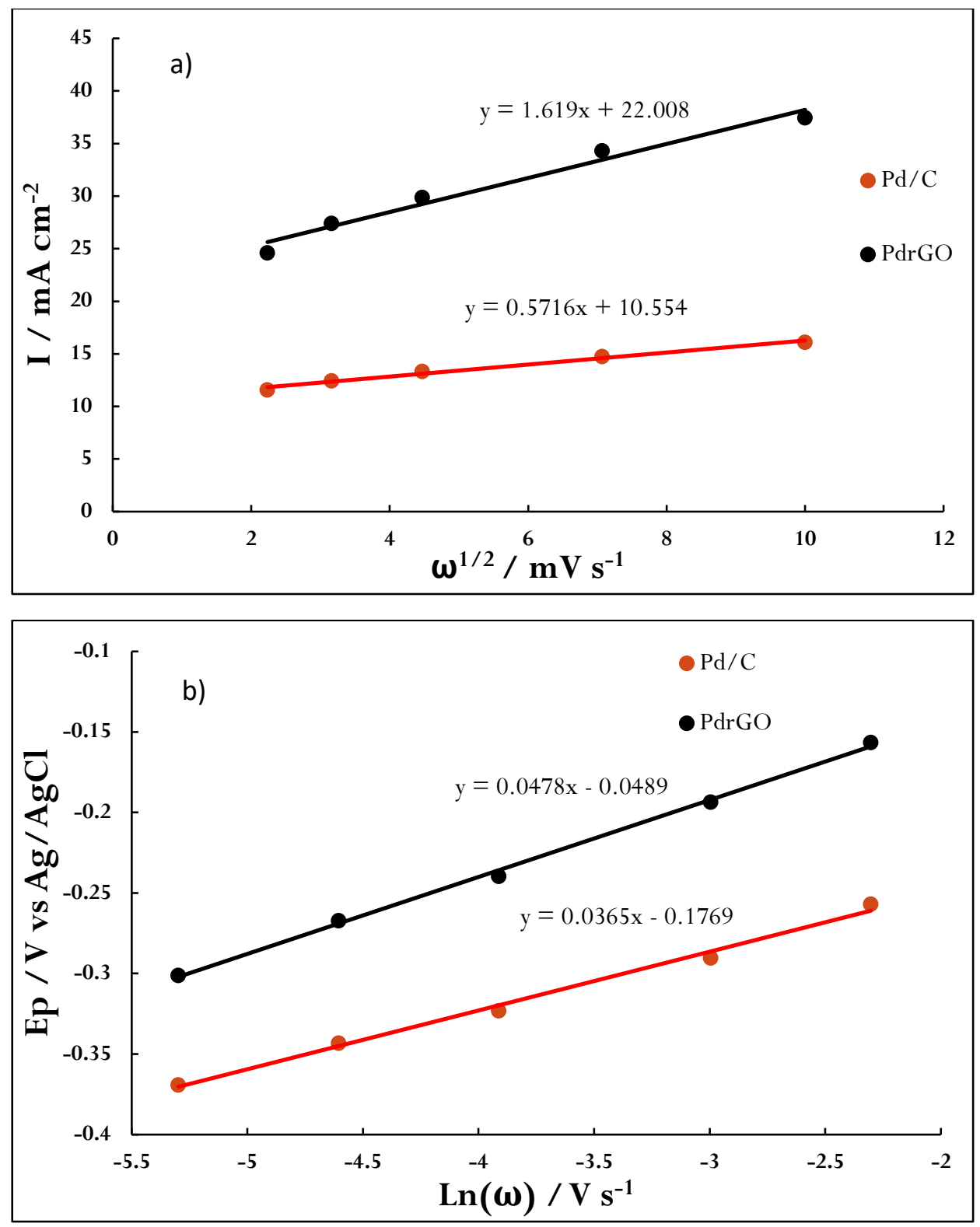

Figure S6. a) Plot of Forward peak current vs sqaure root of scan rate and; b) plot of corresponding potential vs $\ln (\omega)$. 


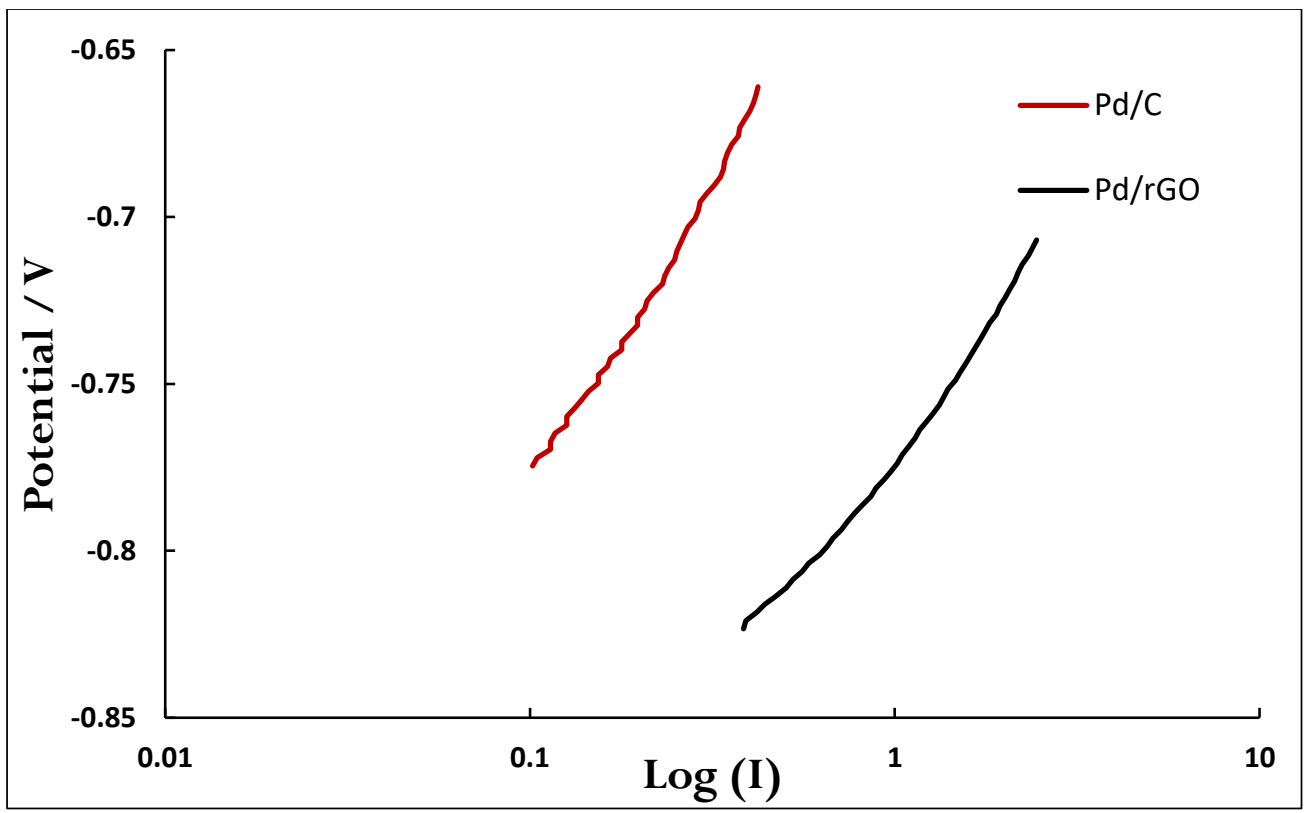

Figure S7: Tafel Plots for the catalyst in $1.0 \mathrm{M}$ HCOONa and $1.0 \mathrm{M} \mathrm{NaOH}$ at $0.5 \mathrm{mV} \mathrm{s}^{-1}$.

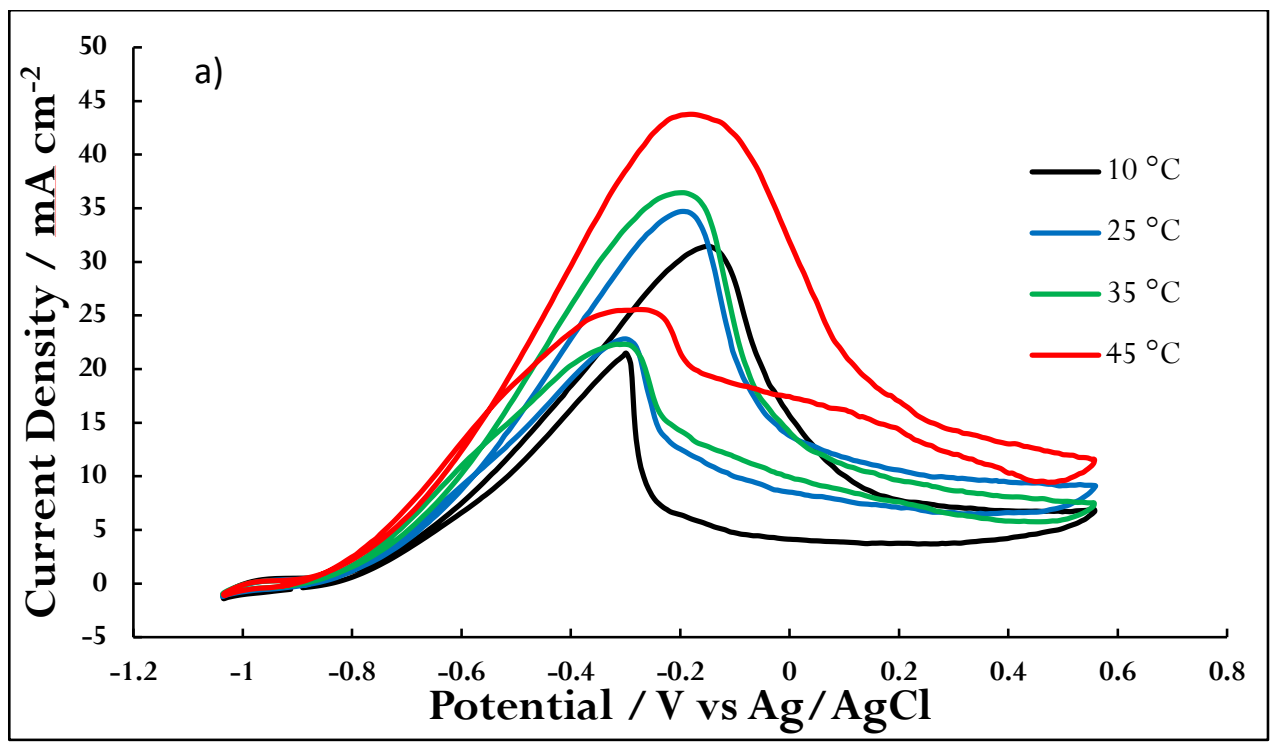



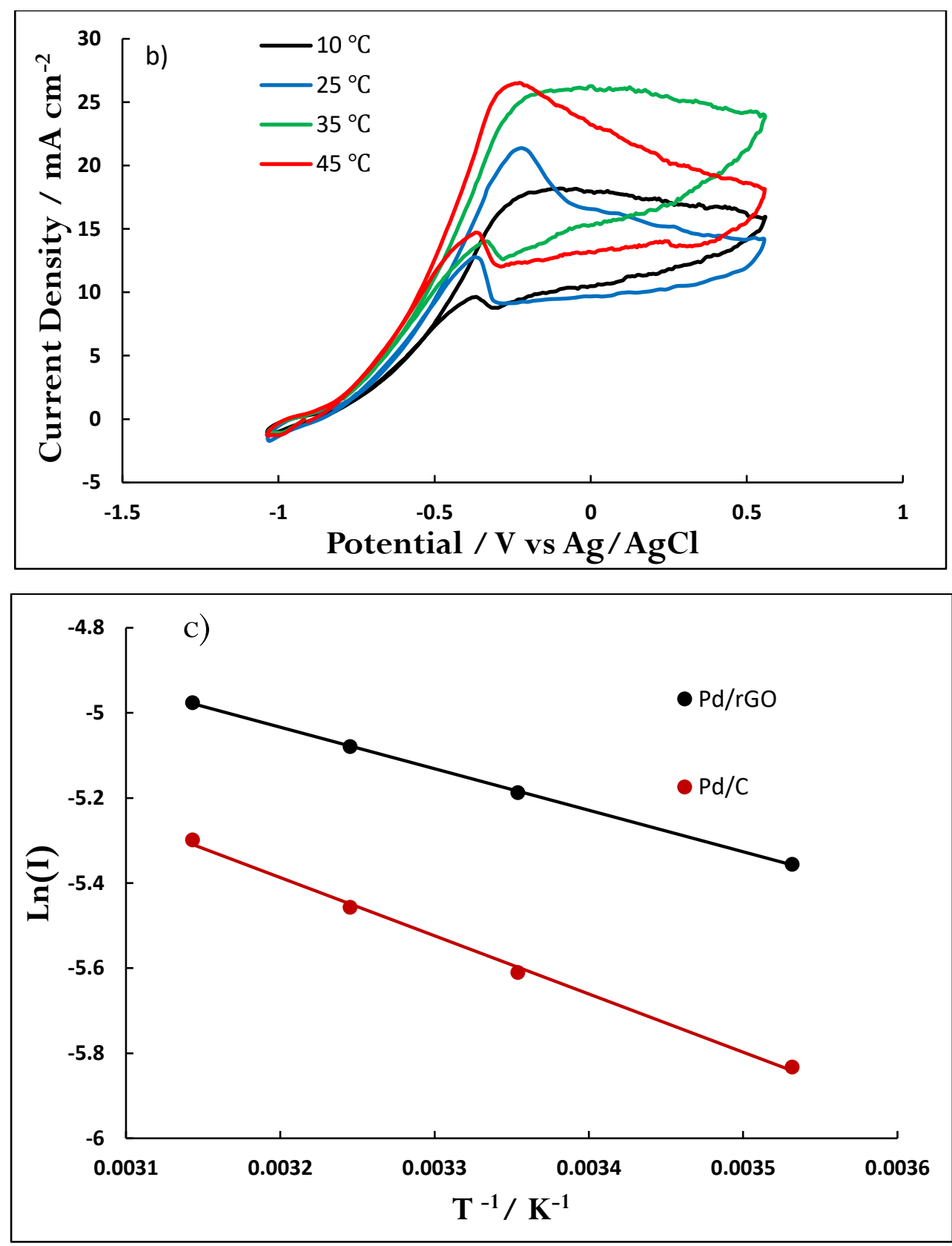

Figure S8. CV scans of the catalysts at different temperatures in $1.0 \mathrm{M} \mathrm{HCOONa}$ and $1.0 \mathrm{M}$ $\mathrm{NaOH}$ for a)Pd/rGO and; b) $\mathrm{Pd} / \mathrm{C}$ at $20 \mathrm{mV} \mathrm{s}^{-1}$ and c) Arrhenius plots for the catalysts. 